\title{
Desmoid Tumor of the Thigh with Multiple Recurrences
}

Roxana Livadariu ${ }^{1}$, Daniel Timofte ${ }^{1^{*}}$, Mihaela Blaj ${ }^{2}$, Delia Ciobanu ${ }^{3}$, Lidia Ionescu ${ }^{1}$ and Radu Dănilă ${ }^{1}$

${ }^{1}$ Department of General Surgery, "St. Spiridon" Hospital, "Gr.T. Popa” University of Medicine and Pharmacy, lasi, Romania

${ }^{2}$ Department of Intensive Care, "St. Spiridon" Hospital, "Gr.T. Popa” University of Medicine and Pharmacy, Iasi, Romania

${ }^{3}$ Department of Pathology, "St. Spiridon" Hospital, "Gr.T. Popa" University of Medicine and Pharmacy, lasi, Romania

“Corresponding author: Daniel Timofte, MD, 3rd Surgical Unit, “St. Spiridon” Hospital lași,Bd. Independenței, No 1, 700111, lași, Romania, Phone: +40 (0) 232 2408 22, E-mail: antimofte@yahoo.com

Received date: 03 December 2013, Accepted date: 30 December 2013, Published date: 15 May 2014

Copyright: (c) 2014 Timofte D, et al. This is an open-access article distributed under the terms of the Creative Commons Attribution License, which permits unrestricted use, distribution, and reproduction in any medium, provided the original author(s) and source are credited.

\begin{abstract}
Background: Desmoid tumors are rare neoplasms of uncertain etiology arising from fascial or deep musculoaponeurotic structures. Although with benign histological appearance and no metastatic potential, desmoid tumors are locally aggressive tumors with a high rate of local recurrence.

Case Report: The case of a 47 years old woman repeatedly operated for a recurrent desmoid tumor of the right thigh is presented. The initial radical excision was followed by adjuvant radiotherapy but local recurrence was diagnosed one year after. The pathology report revealed aspects of desmoid tumor. The patient was reoperated and subsequently referred for chemo- and hormonal therapy. A second recurrence occurred 20 months later and the patient was again operated with microscopic tumor free margins and positive long term outcome.

Conclusion: Desmoid tumors are benign tumors with unpredictable natural history. Best management involves a multidisciplinary approach. Concerning diagnosis, the best approach is performing a MRI examination. Wide surgical resection with adjuvant radiation therapy remains the main treatment option for local control.
\end{abstract}

Keywords: Desmoid Tumor; Surgery; Recurrence

\section{Introduction}

Desmoid tumors are rare neoplasms of uncertain etiology arising from fascial or deep musculo-aponeurotic structures that may occur at any age, but usually in young adults with peak prevalence between 25 and 35 years [1-3]. In adults, the tumor has a predilection in premenopausal women. Incriminated etiological factors are trauma or local surgery history, genetic factors like inherited mutation in APC gene (adenomatous polyposis coli) and high estrogenic states, including pregnancy [4]. Characterized by a benign histological appearance and no metastatic potential, desmoid tumors are locally aggressive tumors with a high rate of recurrence [5]. They may be localized in the abdominal wall, the bowel, and the mesentery (associated with familial adenomatous polyposis) or in extraabdominal sites, such as the trunk and the extremities. The incidence of desmoid tumors ranges from 2 to 4 per million and almost half of them occur in the extremities and trunk [6].

\section{Case Report}

A 47 years old woman was hospitalized in our clinic for a swelling of the upper third of the right thigh, gradually increasing in size in the last 3 months. She had a history of an incomplete tumor excision on the same region, performed in another surgical unit 1 year ago. The pathological report showed at that time a fibroma with an important adipose structure. The local physical examination showed a tumoral mass located on the external face of the right thigh, under an old 10 centimeters long scar. The tumor was $8 \times 4 \mathrm{~cm}$, of firm consistency, painless, relatively fixed and seemed to infiltrate the subcutaneous tissue of the described area (Figure 1).

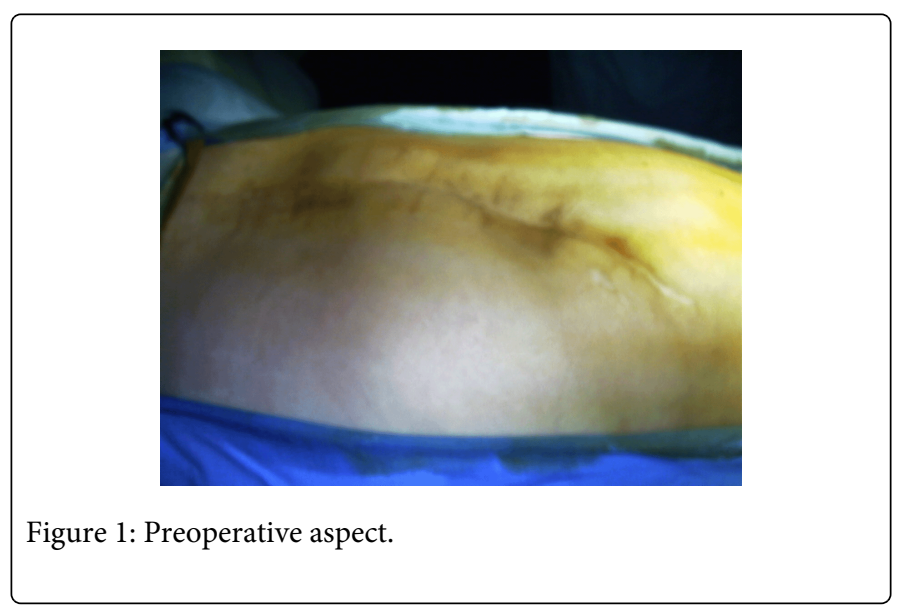

No motor, sensitivity or vascular alterations of the right lower limb were found. The CT scan revealed an expansive irregularly outlined tumoral mass on the upper third of the right thigh and gluteal region topography. The superior extremity of the tumor reached 1 centimeter above the upper bound of the right acetabulum and infiltrated the large and middle gluteal muscles. The tumor also invaded the semitendinosus, semimembranosus and right biceps femoris muscles without bone lesions. Under general anesthesia a wide tumor excision was performed with macroscopic tumor free margins (Figure 2). 


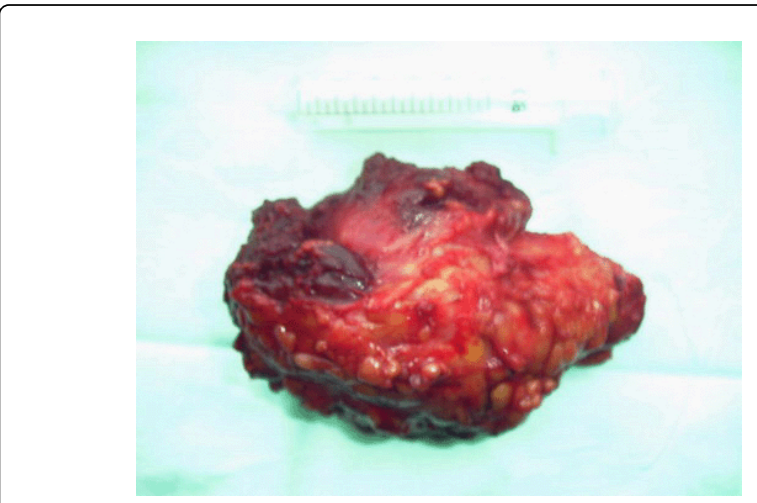

Figure 2: Operative specimen.

The postoperative evolution was uneventful and the patient was discharged after 8 days. The pathology report revealed a desmoid tumor. As recommended by the oncology board, adjuvant radiotherapy was performed. One year later the patient was readmitted for a $6 \times 3 \mathrm{~cm}$ tumor mass, located on the posterior face of the lower third of the same thigh, clinically and radiologically suggestive for a local recurrence. Wide tumor excision was carried out and histological result was again desmoid tumor. The patient underwent for chemotherapy with metothrexate and vinblastine and antihormonal therapy with tamoxifen. After 20 months the patient was hospitalized for a new recurrence showing symptoms and signs of sciatic nerve paresis. The MRI showed an inhomogeneous $5.7 \times 15.6$ centimeters large tumor mass that invades almost entirely the right biceps femoris muscle extending to the vastus lateralis muscle. Superior to this one, the images showed another tumor mass located on the posterior face of vastus lateralis and major gluteal muscle, $5.3 \times 10 \times 3.5$ centimeters large and with imprecise boundaries (Figure 3 ).

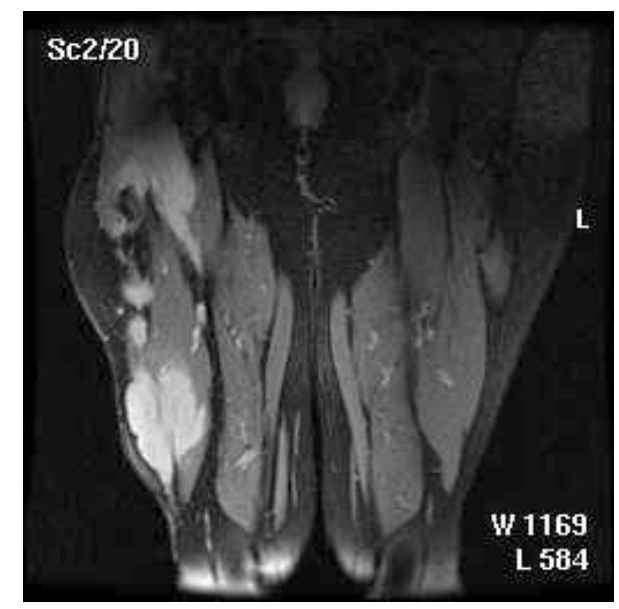

Figure 3: MRI aspect.

The patient was reoperated and a radical surgical excision with microscopically free margins (frozen section examination) was done. The postoperative follow-up showed no signs of recurrence after 6 months.

\section{Discussion}

Although computer tomography shows the extent of the tumor and its relationship to the neurovascular structures, magnetic resonance imaging is the modality of choice for the diagnosis and the evaluation of the tumor extent and the progression of the disease before and after treatment. It may also be helpful in differentiating tumour progression from post-surgical fibrosis [7]. Because multicentric and recurrent lesions tend to occur within the same limb or anatomic region, the MRI scanning of the entire extremity will be done once the diagnosis is made.

As far as the therapeutic options are concerned, surgery is the treatment of choice for extra-abdominal desmoid tumors. Wide excision with free tumor marginal resection is the goal standard of primary treatment. Re-excision for treating the recurrent disease is preferred by most authors, resulting in a cure rate similar to that of the primary surgical resection [8]. However, local control remains difficult. Radiation therapy with doses of $50-60$ Gy is a viable alternative to surgery and a useful adjunct to incomplete resection of primary extra-abdominal desmoid tumors for the control of residual disease [8-11]. Radiation therapy alone can be used where surgery might lead to major morbidity and loss of function as well as for patients who have an inoperable tumor or gross residual disease after operative debulking [9]. The use of antihormonal therapy for the treatment of desmoid tumors is based on epidemiological observations for example, higher incidences of desmoids during and after pregnancy and reports of spontaneous tumor regression after menopause [12]. Studies have shown that virtually all desmoid tumors express nuclear estrogen receptor- $\beta$, but only a small subset of patients respond to antihormonal therapies [13]. Because COX-2 seems to play a role in the pathogenesis of desmoid tumors, treatment with NonSteroidal Anti-Inflammatory Drugs (NSAID) that inhibits COX may be effective [14]. A variety of other NSAID such as indomethacin and sulindac, a long-acting analog of indomethacin were associated with partial and complete responses in several nonrandomized retrospective studies, either alone or in combination with hormonal agents such as tamoxifen [15]. In contrast, in cases of an unresectable, rapidly growing and/or symptomatic and/or life-threatening desmoid tumor, traditional cytotoxic chemotherapy mainly with metothrexate and vinblastine may be the treatment of choice. Loco regional chemotherapy in the form of isolated limb perfusion for patients with locally advanced tumors is another alternative to systemic chemotherapy in patients with limb desmoids. Melphalan and recombinant human tumor necrosis factor- $\alpha$ are used as therapeutic agents with overall response rates of up to $80 \%$ [16]. The recurrence rates after wide local excision is reported as more than $40 \%$, related to section margins, extra-abdominal localization of the tumor and age $[8,17,18]$. The predisposition of aggressive fibromatosis to locally recur is related to its infiltrative nature, the lack of pseudo capsule and possibility of diffusion along muscle fibres and fascial planes which makes it difficult for the surgeon to grossly identify the true extent of disease [19]. This may justify the high recurrence rate of the disease after adequate surgery and may also explain the distant recurrence on the same hip occurred to our patient.

\section{Conclusions}

Desmoid tumors are benign tumors with unpredictable natural history. Best management involves a multidisciplinary approach. Concerning diagnosis, the best approach is performing a MRI 
examination. Wide surgical resection with adjuvant radiation therapy remains the main treatment option for local control.

\section{Conflict of Interests}

Authors have no conflict of interests to disclose.

\section{References}

1. Shields CJ, Winter DC, Kirwan WO, Redmond HP (2001) Desmoid tumours. Eur J Surg Oncol 27: 701-706.

2. Murphey MD, Ruble CM, Tyszko SM, Zbojniewicz AM, Potter BK, et al. (2009) From the archives of the AFIP: musculoskeletal fibromatoses: radiologic-pathologic correlation. Radiographics 29: 2143-2173.

3. Kim SJ, Ha DH, Lee SM, Kang H (2013) Desmoid type fibromatosis in the facet joint of lumbar spine: case report and review of literature. Korean J Radiol 14: 818-822.

4. Honeyman JN, Quaglia MP (2012) Desmoid tumors in the pediatric population. Cancers (Basel) 4: 295-306.

5. Posner MC, Shiu MH, Newsome JL, Hajdu SI, Gaynor JJ, et al. (1989) The desmoid tumor. Not a benign disease. Arch Surg 124: 191-196.

6. Pakos EE, Tsekeris PG, Goussia AC (2005) Desmoid tumours of the extremities and trunk: a review of the literature. Int Orthop 29: 210-213.

7. van Kints MJ, Tjon A Tham RT, Vroegindeweij D, van Erp AJ (1993) Magnetic resonance imaging findings in aggressive fibromatosis. Eur J Radiol 16: 230-232.

8. Papagelopoulos PJ, Mavrogenis AF, Mitsiokapa EA, Papaparaskeva KT, Galanis EC, et al. (2006) Current trends in the management of extraabdominal desmoid tumours. World J Surg Oncol 4: 21.

9. Pritchard DJ, Nascimento AG, Petersen IA (1996) Local control of extraabdominal desmoid tumors. J Bone Joint Surg Am 78: 848-854.
10. Merchant NB, Lewis JJ, Woodruff JM, Leung DH, Brennan MF (1999) Extremity and trunk desmoid tumors: a multifactorial analysis of outcome. Cancer 86: 2045-2052.

11. Duggal A, Dickinson IC, Sommerville S, Gallie P (2004) The management of extra-abdominal desmoid tumours. Int Orthop 28 : 252-256.

12. Ma JH, Ma ZH, Dong XF, Yin H, Zhao YF (2013) Abdominal wall desmoid tumors: A case report. Oncol Lett 5: 1976-1978.

13. Deyrup AT, Tretiakova M, Montag AG (2006) Estrogen receptor-beta expression in extraabdominal fibromatoses: an analysis of 40 cases. Cancer 106: 208-213.

14. Signoroni S, Frattini M, Negri T, Pastore E, Tamborini E, et al. (2007) Cyclooxygenase-2 and platelet-derived growth factor receptors as potential targets in treating aggressive fibromatosis. Clin Cancer Res 13: 5034-5040.

15. Janinis J, Patriki M, Vini L, Aravantinos G, Whelan JS (2003) The pharmacological treatment of aggressive fibromatosis: a systematic review. Ann Oncol 14: 181-190.

16. Kasper B, Ströbel P, Hohenberger P (2011) Desmoid tumors: clinical features and treatment options for advanced disease. Oncologist 16 682-693.

17. Shido Y, Nishida Y, Nakashima H, Katagiri H, Sugiura H, et al. (2009) Surgical treatment for local control of extremity and trunk desmoid tumors. Arch Orthop Trauma Surg 129: 929-933.

18. Dalén BP, Bergh PM, Gunterberg BU (2003) Desmoid tumors: a clinical review of 30 patients with more than 20 years' follow-up. Acta Orthop Scand 74: 455-459.

19. Ramamurthy R, Arumugam B, Ramanandham B (2012) Recurrence patterns and management options in aggressive fibromatosis. Indian $\mathrm{J}$ Surg Oncol 3: 222-227. 